\title{
Rol de la familia en la atención de niños con Trastorno Espectro Autista: Un nexo indisoluble
}

\author{
Role of the family in the care of children with Autism Spectrum Disorder:
} An indissoluble nexus

Jorge Andres Monje Santana. ${ }^{1}$

\begin{abstract}
Introduction. The arrival of a child in the family is a reason for great happiness; But when the child is born with some type of disability, multiple concerns, challenges and uncertainties are generated that are difficult to resolve early. In the case of children with Autism Spectrum Disorders, due to their characteristics, together with their parents' ignorance, it is very complex to attend to them properly Objective. Develop a System of Workshops for the guidance, counseling and support of parents of children with Autism Spectrum Disorders, included in Higher Basic Education. Methodology. The research followed a mixed, descriptive, field approach, with the use of theoretical and empirical methods and techniques such as the survey, interview and documentary review. The sample under study consisted of 4 students with Autism Spectrum Disorder, from two Educational Institutions in the North of the City of Guayaquil. Results. 1. Through the bibliographic review, the importance of the role of the family in the processes of care and educational inclusion of cases with Autism Spectrum Disorders is systematized, highlighting the indissoluble link between the family component and the processes of educational inclusion. 2. A System of 15 Workshops is provided, containing guidelines for the advice and support of parents with children with Autism Spectrum Disorders included in Basic Education. Conclusions. The care and inclusion of students with Autism Spectrum Disorders continues to constitute a great challenge for both the family and educational environment, the solution lies in achieving harmony between the role of the family and the educational institution that receives it; This implies guiding and
\end{abstract}

${ }^{1}$ Colegio Provincia del Carchi, themonje16@hotmail.com, ORCID: 0000-0002-5974-0180 
advising families in a timely manner so that they can offer favorable levels of support to their children and maintain the link between them, school and home.

Keywords: Autism Spectrum Disorders, family, workshop system, educational inclusion

\section{Resumen}

Introducción. La llegada a la familia de un hijo es motivo de gran felicidad; pero cuando el niño nace con algún tipo de discapacidad se generan múltiples preocupaciones, desafíos e incertidumbres difíciles de resolver tempranamente. En el caso de los niños que presentan Trastornos del Espectro Autista, debido a sus características, unidas al desconocimiento de los padres, hace que sea muy complejo atenderlos adecuadamente Objetivo. Elaborar un Sistema de Talleres para la orientación, asesoramiento y apoyo a padres de hijos con Trastornos del Espectro Autistas, incluidos en la Educación Básica Superior. Metodología. La investigación siguió un enfoque mixto, de tipo descriptiva, de campo, con la utilización de métodos teóricos y empíricos y técnicas como la encuesta, entrevista y la revisión documental. La muestra objeto de estudio estuvo compuesta por 6 estudiantes con Trastorno del Espectro Autistas, procedentes de dos Instituciones Educativas del Norte de la Ciudad de Guayaquil. Resultados. 1. Se logra sistematizar mediante la revisión bibliográfica la importancia del rol de la familia en los procesos de atención e inclusión educativa de los casos con Trastornos del Espectro Autista, destacando el nexo indisoluble entre el componente familiar y los procesos de inclusión educativa. 2. Se aporta un Sistema de 15 Talleres, contentivo de orientaciones para el asesoramiento y apoyo a padres con hijos con Trastornos del Espectro Autistas incluidos en la Educación Básica. Conclusiones. La atención e inclusión de los estudiantes con Trastornos del Espectro Autista sigue constituyendo un gran desafío tanto para el medio familiar como educativo, la solución radica en alcanzar armonía entre el rol de la familia y la institución educativa que lo recibe; ello implica orientar y asesorar oportunamente a las familias para que puedan ofrecer niveles de apoyos favorables a sus hijos y mantener el nexo entre estos, la escuela y el hogar.

Palabras clave: Trastornos del Espectro Autistas, familia, sistema de talleres, inclusión educativa.

\section{Introducción}

Desde épocas primitivas y en correspondencia con las diferentes formaciones económicas y sociales que han existido, la familia ha tenido la alta responsabilidad de atender y resguardar la vida de todos sus miembros, con exclusivo interés en los más pequeños. Tal es así, que es considerada la cédula fundamental de la sociedad. Es en la familia donde se forman y amplían los primeros valores, se sientan las bases para el desarrollo integral de la personalidad y es donde se erigen sobre la base del amor, el respeto y la solidaridad los hábitos de convivencia y conducta social. 
En su generalidad cuando a la familia llega un hijo es un gran regalo, siendo este motivo de felicidad, amor y dedicación. Surgiendo la necesidad de lograr una maternidad y paternidad responsable, donde los padres garanticen todas las condiciones para que sus hijos crezcan y se desenvuelvan en armonía y con la calidad requerida. En esta línea de pensamiento se coincide con Zapata (2020), al resaltar la importancia de la maternidad y paternidad responsable. El autor explica que mantener una maternidad y paternidad responsable constituye un deber y obligación de los padres para con sus hijos; alegando que en determinados casos puede llegar a ser transferida a otros miembros de la familia, de forma acordada o impuesta pero siempre se parte de considerar que la custodia y cuidado del menor, es esencial para su vida y nada puede eximirnos de esta responsabilidad.

Ahora bien, cuando nace un niño con algún tipo de discapacidad se generan en el seno familiar múltiples preocupaciones, desafíos e incertidumbres difíciles de resolver por parte de los padres y demás miembros del núcleo familiar. En el caso de los niños que presentan Trastornos del Espectro Autista (TEA), lo anterior es extremadamente complejo; justamente por las características que manifiestan, fusionadas a la falta de conocimientos y preparación de los padres para atenderlos adecuadamente, de ahí que sea importante encontrar alternativas que de forma urgente permitan orientar a los padres en todo lo relacionado con el rol a desempeñar ante la llegada de un niño con Trastorno del Espectro Autista, cabe preguntarnos: ¿Cómo influye el rol que desempeña la familia en la atención a niños que tienen Trastorno Espectro Autista que se encuentran incluidos en la Educación Básica Superior? Para dar solución a lo expuesto la presente investigación reconoce como Objetivo General: Elaborar un Sistema de Talleres para la orientación, asesoramiento y apoyo a padres de hijos con Trastornos del Espectro Autistas incluidos en la Educación Básica Superior.

\subsection{Orígenes, Conceptualización y Clasificación. Formas de intervención con los estudiantes que presentan TEA.}

El análisis y sistematización de las teorías antecedentes relacionadas con el término Autismo, confirman que estamos en presencia de un tema de gran actualidad e importancia; sin embargo independientemente de las variadas investigaciones realizadas afines con el Autismo, aún persisten brechas epistémicas y metodológicas sin resolver, provocando la necesidad de continuar profundizando en el tema. Dentro de estas investigaciones o brechas por resolver las que se dirigen al rol de la familia en los procesos de atención e inclusión educativa de los niños con TEA revisten particular interés. Es por ello que en primer orden intentaremos tener un acercamiento a los orígenes, conceptualización, formas de clasificación e intervención que se han venido dando desde épocas anteriores sobre el TEA hasta la actualidad.

En esta línea de pensamientos resulta transcendental antes de conceptualizar qué se entiende por Trastorno del Espectro Autista, comprender el origen del término Autismo. 
Justamente en este aspecto especial utilidad tienen los estudios realizados por Figueredo, (2011), Piro (2017), seguidos por Yerovi (2021), los cuales señalan que este término provine del griego $\alpha \hat{\tau} \tilde{\tau}$ - el cual indica "que actúa sobre sí mismo o por sí mismo", mientras que el sufijo -ismo, lo relacionan con cierto tipo de tendencia a la discapacidad.

En la búsqueda y análisis de la bibliografía precedente se ha podido determinar que para la comunidad científica, el punto de inicio de mayor significatividad en cuanto a la comprensión y definición del Autismo lo tienen los estudios de Leo Kanner, (1943) y Hans Asperger en (1944). Ambos autores son considerados los precursores en abordar esta temática. Sus aportes marcan el reconocimiento y explicación de muchas interrogantes, sintomatologías y características que hasta esa fecha para la ciencia aparecían de manera inconclusa, sentándose de este modo las bases para la realización de estudios posteriores. Tanto Kanner como Hans, logran establecer dentro de los signos más característicos presentes en las personas autistas, las tocantes con los trastornos del desarrollo y la identificación de las áreas más afectadas: lenguaje, comunicación y comportamiento.

Si bien es cierto, que tanto Kanner como Hans en su tiempo logran concretar y revolucionar ideas muy valiosas y de gran alcance respecto al Trastorno del Espectro Autista, es meritorio reconocer también que otros investigadores e instituciones han dado seguimiento y pasos importantes en este tema.

Obsérvese como en el Manual de Diagnóstico y Estadística de Trastornos Mentales (DSM-IV 1992), se incluye el autismo, en la categoría de Trastornos Generalizados del Desarrollo. Clasificando este trastorno en alto, medio y bajo nivel de funcionamiento. Nótese también como la American Psychiatric Association. DSM, (2018), describe las diferentes etapas por el cual este término ha venido atravesando, determinando 5 etapas, comprendidas entre 1952 hasta llegar al año 2013, donde establece una sola categoría denominándolo: Trastorno del Espectro Autista.

En el (2019), la National Institute of Mental Health (NIH), plantea que el Trastorno del Espectro Autista (TEA), es un trastorno del desarrollo que afecta la comunicación y el comportamiento. Describe que se trata de un "trastorno del desarrollo", el cual puede ser diagnosticado a cualquier edad pero preferiblemente debe tratarse desde edades tempranas dada la gama y diversidad de sintomatología, variedad de características y grado en que se presenta. Es por ello que lo denominan: Espectro.

Atendiendo a todo lo expresado la Real Academia Española, (2020), indica que el término Autismo se vincula e identifica con dos aspectos fundamentales:

1. Repliegue patológico de la personalidad sobre sí misma.

2. Trastorno del desarrollo que afecta a la comunicación y a la interacción social, caracterizado por patrones de comportamiento restringidos, repetitivos $\mathrm{y}$ estereotipados. 
Las diferentes literaturas y fuentes consultadas nos llevan a concordar con lo planteado por: Riviére, \& Martos, (2000), Figueredo, (2011), Jones (2017), Tendlarz, (2018), Jara, (2019), Yerovi, (2021) y otros autores al considerar que se trata de un trastorno profundo del desarrollo, el cual desde su detección ha provocado y sigue provocando mucha contradicción en la comunidad científica, lo cual hace que sea un gran desafío para los especialistas de las diferentes ramas y por supuesto para los padres de familia, que requieren de sistemas de orientaciones y ayudas que les permitan acortar la grieta entre su hijo y el mundo que les rodea.

Es meritorio señalar que los aportes y estudios realizados sobre el tema dejan claro que a pesar de la incertidumbre aún existente sobre los aspectos por explicar, ha quedado demostrado que el Autismo no es una enfermedad, sino un trastorno muy complejo que precisa de formas de intervención multidisciplinares, oportunas y diversas. Solo así se logrará dar respuesta a las necesidades de los niños con TEA. Para ello urge trabajar de conjunto con el medio sociofamiliar y educativo, fomentando en los niños y padres la aceptación, el amor y la realización de actividades que propicien el desarrollo integral del niño independientemente a su condición.

\subsection{El rol de la familia en el proceso de inclusión educativa de los niños con Trastornos del Espectro Autista.}

Si consideramos las escalofriantes estadísticas que expresan que tanto a nivel internacional como nacional el índice de niños con TEA ha ido en aumento, llegando a señalarse por parte de la Organización Mundial de la Salud, que uno de cada 160 niños tiene Trastorno del Espectro Autista (TEA), estaremos justificando aún más la pertinencia e importancia del tema. Cabe preguntarnos entonces ¿Están las familias preparadas para afrontar el reto de la crianza, educación e inclusión de los niños con TEA?.

A la primera interrogante se suceden otras más que de acuerdo con Rodríguez (2014), los padres y educadores se preguntan a diario ¿Qué es lo que debo hacer? ¿Cómo se debe trabajar con las familias? ¿Cómo influirnos y ayudarnos mutuamente familia y docente? ¿Cómo incidir en el proceso educativo del niño a partir de nuestro trabajo conjunto?

Por supuesto que las respuestas a estas interrogantes van a depender de considerar diversos factores, desde los que tienen que ver con factores culturales, políticos, sociales, económicos e incluso los valores humanos y la diversidad de modos de familias existentes en la actualidad.

Por ejemplo los aportes López \& Larrea , (2017) señalan que en Ecuador se reportó en el 2016, 1258 personas diagnosticadas con algún Trastorno del Espectro Autista, de los cuales muchos viven en condiciones precarias y con familias disfuncionales.

En correspondencia con las ideas de Baña (2015), se sostiene la importancia que tiene el rol familiar en la calidad de vida de las personas con TEA. Concordamos con el autor en que educar a las personas con TEA implica considerar que el proceso de atención 
educativa debe ofrecérseles a largo de su vida familiar y a su vez permitirle su inclusión socioeducativa.

En este particular Luzuriaga (2017), Patiño (2018), Buenaño y Montero (2019), enfatizan en la necesidad de la participación familiar en los procesos de atención e inclusión de los niños con TEA. Destacan que entre los beneficios de una buena relación entre los padres y los entes educativos se encuentran la mejora del rendimiento académico de los estudiantes, el incremento de la atención de los infantes y la construcción de espacios donde se refuerzan valores como la confianza, responsabilidad y cooperación; todo lo cual influye de manera positiva en el aumento de la autoconfianza en los niños y en el reconocimiento de sus propias potencialidades.

En el caso de Ecuador resultan escasas las investigaciones que aporten soluciones concretas que permitan enriquecer el nivel de preparación de las familias para poder atender adecuadamente a los hijos con TEA .Es una realidad que muchos padres se sienten desconcertados y desorientados acerca de cómo enfrentar la educación e inclusión de sus hijos, mucho más cuando estos tienen una condición diferente a la norma general bien sea por déficit o por cualquier otra razón.

Atendiendo a esto se ha considerado que la sistematización de las teorías precedentes realiza un abordaje al tema del autismo pero aun persisten limitaciones en cómo perfeccionar el rol de la familia para facilitar los procesos de atención e inclusión de los niños con TEA a la Educación Básica Superior.

\section{Metodología}

La investigación siguió una metodología con enfoque mixto, de tipo descriptiva, de campo, acompañada del empleo de métodos teóricos y empíricos y técnicas como la encuesta, entrevista y la revisión documental, todo lo cual facilitó el desarrollo del proyecto sobre bases científicas. La muestra objeto de estudio estuvo compuesta por 4 estudiantes con Trastorno del Espectro Autistas, ubicados en dos Instituciones Educativas del Norte de la Ciudad de Guayaquil. Para su determinación se tuvieron en cuenta como criterios de inclusión:

- Estar diagnosticados con Trastornos del Espectro Autista.

- Presentar dificultades para acceder a los procesos de inclusión educativa

- Contar con el apoyo y disposición familiar

- Estar escolarizados en la Educación Básica Superior

- Tener entre 12 y 14 años de edad.

\section{Resultados}

Una vez analizados los resultados derivados de la aplicación de los instrumentos de investigación se logra como principales resultados los siguientes: 
$\checkmark$ En armonía con lo inicialmente expuesto, el estudio sistematiza los posicionamientos teóricos que sustentan el nexo e importancia del rol familiar en los procesos de atención e inclusión educativa de los casos con Trastornos del Espectro Autista. Coincidiéndose con autores precedentes tales como: Baña, (2015), Luzuriaga (2017), Patiño (2018), Buenaño y Montero (2019), quienes reafirman la importancia del rol familiar en los procesos de inclusión y atención de los niños con TEA, precisamente porque independientemente a las investigaciones realizadas aún este tema sigue constituyendo un desafío tanto para los padres como para el personal que labora con los niños.

$\checkmark$ Se aporta un Sistema de 15 Talleres contentivo de orientaciones para el asesoramiento y apoyo a padres de hijos con Trastornos del Espectro Autistas incluidos en la Educación Básica Superior, los cuales se encuentran compuestos por título, objetivos, tiempo, recursos y actividades. Dichos talleres siguen una lógica organizativa para su desarrollo la cual incluye tres fases: La primera se dirige a la concientización sobre la importancia de la participación de los padres en la atención a los niños con TEA, la segunda fase de los talleres se centra en la capacitación a los padres sobre las situaciones que de manera cotidiana pueden presentar sus hijos y la tercera fase ofrece una serie de actividades para la interacción de docentes y padres de familia en la atención de niños con TEA

$\checkmark$ Destaca entre los beneficios fundamentales el aporte de orientaciones, asesoría y apoyos para el perfeccionamiento del rol familiar como componente esencial para el proceso de inclusión educativa de los estudiantes con Trastornos del Espectro Autista.

$\checkmark$ La implementación del Sistema de Talleres podrá favorecer de manera oportuna el rol familiar de los padres con hijos que presentan Trastornos del Espectro Autista. 
Figura No 1. Fases Organizativas para el Desarrollo del Sistema de Talleres de Orientación Familiar.
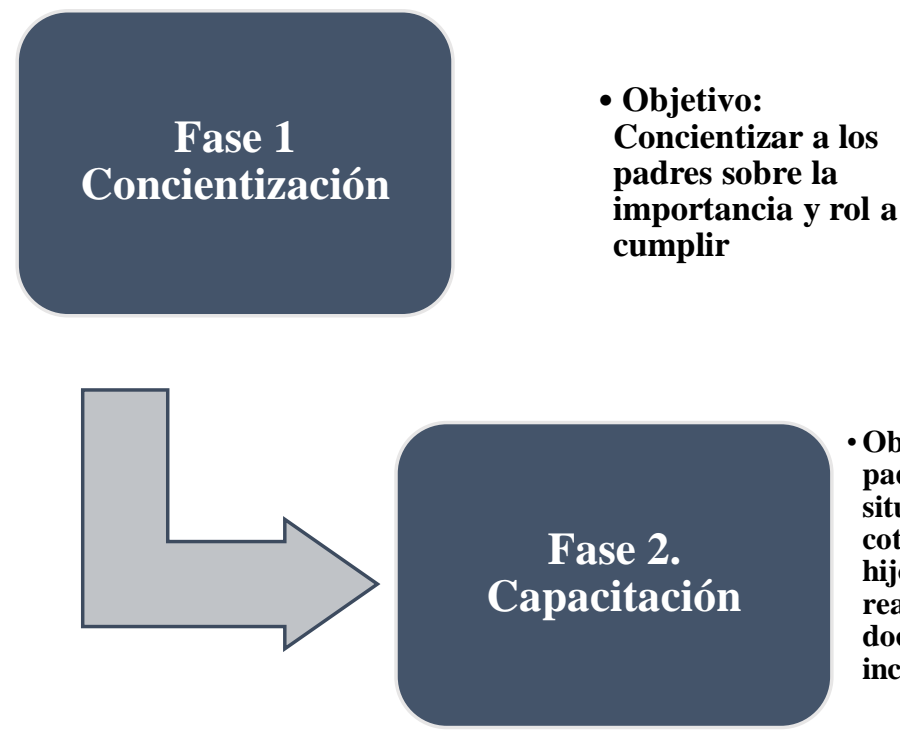

- Objetivo: Capacitar a los padres sobre las diferenteslas situaciones que de manera cotidiana pueden presentar sus hijos $\mathrm{y}$ las actividades a realizar d econjunto con los docentes para propiciar la inclusipn educativa

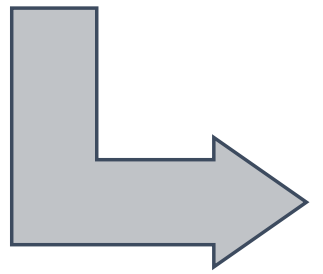

Fase 3. Aplicación de Actividades de Interacción entre Docentes y Padres

Elaborado por: Monje (2021)

\section{Conclusiones}

A modo de conclusión se considera que:

- La atención e inclusión de los estudiantes con Trastornos del Espectro Autista sigue constituyendo un gran desafío tanto para el medio familiar como educativo, la solución radica en alcanzar armonía entre el rol de la familia y la institución educativa que lo recibe; ello implica orientar y asesorar oportunamente a las familias para que puedan ofrecer niveles de apoyos favorables a sus hijos y mantener el nexo entre estos, la escuela y el hogar.

- El Sistema de Talleres propuesto una vez aplicado podrá contribuir a perfeccionar significativamente el rol familiar de los padres con hijos con Trastornos del Espectro Autista, facilitando los procesos de inclusión y atención a la diversidad que se manifiesta según cada caso.

\section{Referencias Bibliográficas}

American Psychiatric Association.DSM. (OCTUBRE de 2018). Manual de diagnóstico estadístico de desórdenes mentales DSM_V.Amer.Psychiatric. Obtenido de 
DMS-5

Actualización:

https://psychiatryonline.org/pbassets/dsm/update/DSM5Update_octubre2018_es. pdf

Baña, M. (2015). El rol de la familia en la calidad de vida y la autodeterminación de las personas con Trastorno del Espectro del Autismo. Ciencias Psicológicas, 9(2), 323- 336. Departamento de Psicología Evolutiva y de la Educación. Universidad de Coruña. España. Consultado el 28 de Abril dl 2020. Obtenido de https://www.redalyc.org/articulo.oa?id=459545411009

Buenaño, L. Montero, R. (2019) El rol de la familia en la inclusión educativa en niños 4 a 5 años. Talleres para representantes legales. Repositorio de la Universidad de Guayaquil. Obtenido de http://repositorio.ug.edu.ec/bitstream/redug/45951/1/BPARV-PEP-19P066.pdf

DSM-IV., (1992) Manual de Diagnóstico y Estadística de Trastornos Mentales, 4ta. Ed., Asociación Norteamericana de Psiquiatría, EUA.

Figueredo, L., (2011). Metodología para la atención educativa de niños con TEA de bajo nivel de funcionamiento Tesis en Opción al Grado Científico de Doctor en Ciencias de la Cultura Física. La Habana, Cuba.

Jara, M., (2019). Comorbilidades clínicas en niños y niñas autistas diagnosticados en el año 2015 en Quito y Guayaquil. Obtenido de http://repositorio.puce.edu.ec/handle/22000/16460

Jones, I., (2017). Estrategia educativa para estimular el desarrollo de la comunicación y el lenguaje en niños con Trastorno del Espectro Autista del centro de salud el cisne II. (U. L. Rocafuerte, Ed.) Obtenido de http://repositorio.ulvr.edu.ec/handle/44000/2947

Kanner, L., (1943). Trastornos autistas del contacto afectivo. Obtenido de http://espectroautista.info/kanner.html

Luzuriaga, L. (2017) La educación a la familia para apoyar el aprendizaje de niños con discapacidad intelectual de la unidad educativa "ciudad de esmeraldas. Repositorio ULVR. Obtenido de http://repositorio.ulvr.edu.ec/bitstream/44000/2874/1/TM-ULVR-0021.pdf

López \& Larrea, (2017). Autismo en Ecuador: Un grupo social en espera de atención. Rev Ecuat Neurol vol.26 no.3 Guayaquil sep./dic. 2017, 12. Obtenido de http://scielo.senescyt.gob.ec/scielo.php?script=sci_arttext\&pid=S263125812017000200203.

National Institute of Mental Health (NIH). (2019). htt// www.nimh.nih.gov. Obtenido de https://www.nimh.nih.gov/health/publications/autismspectrumdisorder/index.shtml. 
Patiño, C. (2018) El entorno familiar y su influencia en el control de emociones de niños con autismo leve en las edades de 3 a 5 años de la unidad educativa particular "tejar". Repositorio ULVR. Obtenido de http://repositorio.ulvr.edu.ec/bitstream/44000/3107/1/TM-ULVR-0118.pdf

Piro, M.C., (2017). El Autismo Perspectiva Teórico clínicas y desafíos contemporáneos. Obtenido de Portal de Libros UNLP: https://libros.unlp.edu.ar/index.php/unlp/catalog/book/836

Real Academia Española. (2020). Diccionario de la Lengua Española. Obtenido de DEL: https://dle.rae.es/autismo.

Riviére, A \& Martos, J. (2000). El pequeño niño con autismo. Madrid: Editorial Instituto de Migraciones y Servicios Sociales

Rodríguez, M. (2014). Trabajo conjunto familia y docente, el caso de un padre con su hijo. Revista Electrónica Educare (Educare Electronic Journal), 297-309. Escuela Nuestra Señora de Lourdes Heredia, Costa Rica. Obtenido de file:///C:/Users/Invitado1/Documents/LIBROS\%20PARA\%20LA\%20TESIS\%2 0DEL\%20ROL\%20DE\%20LA\%20FAMILIA\%20Y\%20SU\%20TRASTORNO $\%$ 20ESPECTRO\%20AUTISTA/revista\%20electronica\%20Trabajo\%20conjunto $\% 20$ familia\%20y\%20docente,\%20e1\%20caso\%20RODRIGUZ\%20MARIA.pdf

Tendlarz, S., (2018). La dirección de la cura en el autismo y en la psicosis en la Infancia. Obtenido de X Congreso Internacional de Investigación Práctica Profesional en Psicología. MERCOSUR Universidad de Buenos Aires: https:// www.aacademica.org/000-122/554

Yerovi, MC., (2021), Competencias docentes para la detección temprana del Trastorno del Espectro Autista en niños de preescolar. Tesis en Opción al Título de Magister en Educación. Mención Inclusión y Atención a la Diversidad. Universidad Laica Vicente Rocafuerte, Obtenido de http://repositorio.ulvr.edu.ec/bitstream/44000/4252/1/TM-ULVR-0268.pdf

Zapata, A. (2020). Maternidades y paternidades transnacionales: una reflexión desde los procesos de interacción mediada. Revista Colombiana de Sociología, 43(1), 81107. Consultado de file:///C:/Users/LT/Downloads/78954-449838-3-PB.pdf

\section{LCiencia}




\section{PARA CITAR EL ARTÍCULO INDEXADO.}

Monje Santana, J. A. (2021). Rol de la familia en la atención de niños con Trastorno Espectro Autista: Un nexo indisoluble. Explorador Digital, 5(3), 6-16. https://doi.org/10.33262/exploradordigital.v5i3.1749

\section{LCiencia}

El artículo que se publica es de exclusiva responsabilidad de los autores y no necesariamente reflejan el pensamiento de la Revista Explorador Digital.

El artículo queda en propiedad de la revista y, por tanto, su publicación parcial y/o total en otro medio tiene que ser autorizado por el director de la Revista Explorador Digital.
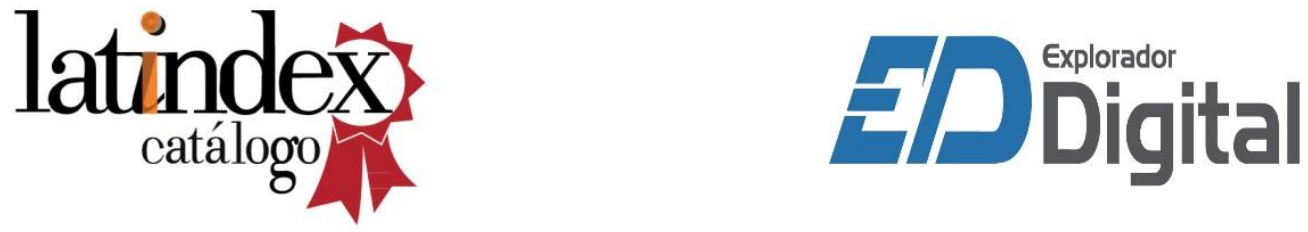